\title{
Decibel Imaging for Generating Medical Report using Ultrasonic
}

\author{
Sathya.N, Sanjai.D, Swathi.R
}

\begin{abstract}
Medical imaging is commonly used for diagnosis and care in clinical practice. Report-writing would be prone to mistakes for inexperienced physicians, and experienced physicians would be time consuming and boring. To handle these issues, we study the automated generation of medical imaging reports. This task presents several challenges. First, a complete report contains multiple heterogeneous types of information including findings and tags. Second, abnormal regions in medical images are difficult to spot. Third, usually, the reports are lengthy and contain multiple sentences. To deal with these challenges, we (1) build a multi-task learning framework which jointly performs the prediction of tags and therefore the generation of paragraphs, (2) propose a co-attention mechanism to localize regions containing abnormalities and generate narrations for them, (3) develop a hierarchical LSTM model to get long paragraphs. We show the efficacy of the proposed methods on two datasets which are publicly accessible.
\end{abstract}

Keywords: Medical Imaging, Long Short Term Memory (LSTM), Ultrasound Imaging.

\section{INTRODUCTION}

Ultrasound imaging plays a key role in medical imaging because of its non-invasive nature, low cost and real-time imaging capabilities. Using ultrasonic (aka ultrasound) waves at a maximum of 3 to $20 \mathrm{MHz}$, scientific ultrasonic imaging is achieved. Ultrasound waves are created from the transducer and pass across body tissues, and it is mirrored back when the wave hits an area or surface of specific structure or acoustic design. The device (the transducer array) absorbs these echoes and they are converted into electric current. The image generated using Ultrasound screening - generally referred to as ultrasound scanning-is labeled ultra sonogram. It is the procedure and method of producing visual depictions of a body's interior for clinical examination and surgical action, and visual depiction of the role of those organs or tissues (physiology). Medical imaging helps to expose internal mechanisms that are concealed by the skin and bones, and to detect and cure illness. Medical imaging also provides a record of natural anatomy and physiology such that irregularities can be detected.

Revised Manuscript Received on April 27, 2020.

*Correspondence Author

Sathya.N*, Department of Information Technology, Sri Shakthi Institute of Engineering and Technology, Coimbatore, India. Email: nsathyait@siet.ac.in

Sanjai.D, Department of Information Technology,Sri Shakthi Institute of Engineering and Technology, Coimbatore, India. Email: sanju29111998@gmail.com

Swathi.R, Department of Information Technology,Sri Shakthi Institute of Engineering and Technology, Coimbatore, India. Email: swathirmkd@gmail.com

(c) The Authors. Published by Blue Eyes Intelligence Engineering and Sciences Publication (BEIESP). This is an open access article under the CC BY-NC-ND license (http://creativecommons.org/licenses/by-nc-nd/4.0/)
Although imaging of extracted organs and tissues may be performed for medical purposes, these procedures are generally considered part of the pathology rather than part of the Medical imaging.The classification of the collection of techniques that noninvasively generate representations of the internal dimension of the body is also interpreted. Medical imaging can be used in this limited context as the application to quantitative inverse problems. This assumes cause (the characteristics of living tissue) is derived from impact (the signal observed). In the case of medical ultrasound, the probe consists of waves and ultrasonic pressure echoes that go through the tissue to reveal the internal structure. The picture quality would be improved with the usage of higher frequencies but this at the same time reduces the penetration range. There are numerous ultrasonic imaging types. The most popular modes are (a) b-mode - simple two-dimensional strength mode, (b) mmode - to determine moving body parts (e.g. heart movements) from replicated speech, and (c) color modepseudo-coloring via Doppler detection based on the observed cell activity.

\section{RELATED WORKS}

In this section is addressed the relevant research which is focused on the question expected by this project.

In paper [6], K Thangavel, $\mathrm{R}$ Manavalan,I Laurence Aroquiaraj, B-Scan pictures are used for the characterisation of the tissue. Such pictures are collected employing a basic linear or sector-scanning US microscope, revealing a granular appearance called speckle. Speckle is modeled as a signal-dependent noise, thus appearing to decrease image quality and contrast. The diagnostic properties of the US image modality. Scientists also evolved numerous methods of speckle reduction over a period of your time, but failed to constitute a scientific approach that takes under consideration all the constraints. This dissertation deals with Wiener filtering in wavelet domain as a scientific technique with simple thresholding. The author [7] Jakia Afruz, Va'Juanna Wilson, Image enhancement is used in digital image processing to produce a more robust look to a picture. Color is one among the simplest options for visually enhancing an picture. Pseudocolor refers topainting a picture by converting the gray-scalevalues to a color space in three dimensions. during this Paper We used a frequency-domain pseudocolor technique to boost ultrasonic pictures. to try and do so we've got used three separate forms of transformations which are the transformation of Fourier,

\section{Published By:}

Blue Eyes Intelligence Engineering

\& Sciences Publication

(C) Copyright: All rights reserved. 
the transformation of distinct cosine and therefore the transformation of Walsh-Hadamard. After obtaining those pseudo-color images, we added a high frequency focus filter or histogram stretch as a post phase. During this paper we want to analyze photos via a contextual analysis. Next, we compared pseudo-color images to their original monochrome images. Second, we contrasted each of the three distinct transform shapes. Finally, we contrasted the methods used for post-production. By [8] The author Savita Gupta introduces a unique speckle reduction approach focused on soft thresholding of the logarithmically transformed medical ultrasound signal wavelet coefficients. The approach is predicated on generic simulation of subband coefficients within the Gaussian distributed (GGD). The suggested approach for denoising natural image is developed within the Bayesian context. this is often adaptive to the dimensions since the parameters won't to approximate the edge rely upon data from the dimensions and therefore the sub band. the edge is decided by the noisefree image, where $\mathrm{K}$ is that the variance of the noise and therefore the sub band data of the noise-free image, and $\mathrm{K}$ could be a scale parameter, respectively. Experimental findings indicate that the proposed approach performs better than the median filter and therefore the homomorphic Wiener filter, particularly in terms of retaining features for better diagnosis as needed within the processing of medical images. The author [9] Zhao Chunhorg Digital methods for collecting and storing images play a really significant function in modern medical diagnostics. Photos of live organisms are obtained using multiple techniques like $\mathrm{X}$ ray, ultrasound, computerized tomography (CT), resonance imaging (MRI), and so on. Throughout the retrieval phase, defects within the photos may arise which might adversely impact the interpretation centered on such photos. It highlights the worth of implementing sophisticated digital image processing techniques to boost accuracy by eliminating the noise components present within the picture acquired to assist diagnosis.

By [10] The aurthor Chedsada Chinrungrueng, Aimamorn Suvichakorn, the creation of fetal ultrasonic photos is clarified by segmentation. the newest paper research discusses the creation of enhancement and segmentation algorithms, since the evaluation of the ultrasound is strongly reliant on data quality. Established algorithms section the foetus and permit the anatomical form to be analyzed. this enables the obstetricians a simple screening method to check, and therefore the results will be retained for future research within the database.

\section{Drawbacks of existing system}

- The quality of healthcare is comparatively low

- Writing medical-imaging reports is demanding

- Writing imaging reports is tedious and time consuming

- Radiologist may have to read hundreds 2578 of radiology images per day.

- Typing the findings of every image into computer takes about 5-10 minutes, which occupies most of their working time.

\section{IMPLEMENTATION}

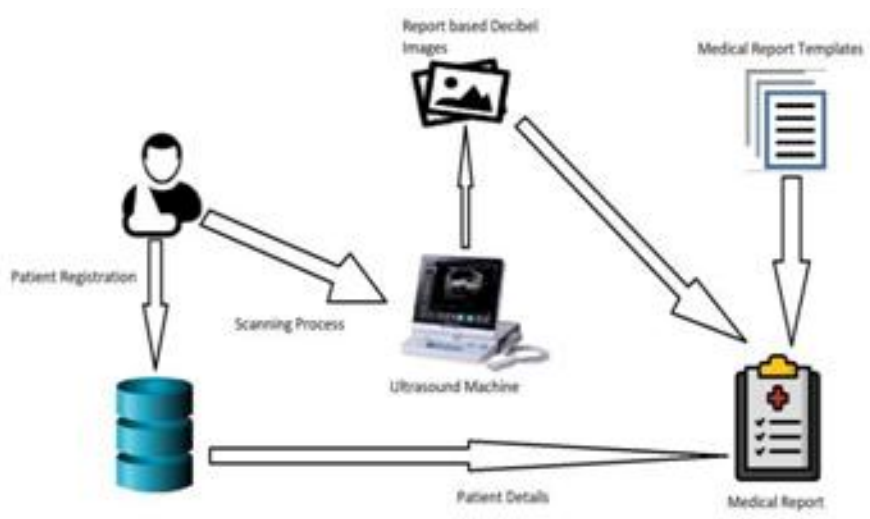

Fig. 1. System Architecture

\section{A. Login-Admin:}

Admin module helps device administrator to set up machine backend and execute simple device setup, mainly specifying predefined drop-down areas, specifying the time schedule classes, etc. The new packages and packs of deals as well as different rates and product categories are specified here for courses, new subjects provided, etc.

Part of the admin configuration is account control that lets users configure connections to a single or multiple division with level / roles definable connections. Admin can also configure overall system security settings such as password power, inactive session time out, inactive user lockout, password reset date, etc. Audit log is a vital aspect of protection-all device modifications are recorded here and it's simple to verify who changed / removed what, at what point, what was the original value, and what is the current value collection.

\section{B. Patient Detail Registry:}

The register module offers a systematic mechanism for entering patient data in a mannerthat: encourages data entry \& consistency by linking Transparent entry to data source, conveniently links back to individual medical information to attach patient data information, and gathers data elements to allow improved management of care programs.

\section{Scanning:}

A digital image capturing module assembly and production process is suggested using a digital image capture module assembly from a photosensitive printed circuit board and a lens holder. The suggested assembly method is characterized by applying a light-impenetrable reinforcing plate to the photosensitive printed circuit board to have both a light-impenetrable impact and a reinforcing effect on the photosensitive printed circuit board, which allows the enclosed photosensitive device to be shielded from side light and backlight, The photosensitive printed circuit board may also be strengthened with structural protection that can avoid deformation or cracking during manufacture and transport. This functionality helps the finished output of the digital image capture module to be more confident of the 
optical efficiency of the captured images during actual operation and thus allows the manufacture of the captured images during production to provide a strong yield rate.

\section{Database Module}

SQL databases like Ignition are the simplest, cheapest, and most versatile way to access them. It also provides a basic graphical user interface named the Database Editor which is used in database tables to create / alter / drop database tables and view / query / edit the details.

\section{PROPOSED WORK}

That motivates us to examine if medical image reports can be produced automatically. We ought to overcome many problems.

Firstly, several heterogeneous sources of knowledge compose a full diagnostic study. When seen in Figure 1, the chest $\mathrm{x}$-ray summary includes a sentence description, observations that are an article, and marks that are a keyword chart. It is technically challenging to produce the heterogeneous knowledge in a single system. We address this issue by developing a multi-task system that treats tag estimation as a multi-label labeling process and treats the production of long explanations as a task of text production. Second, it's difficult how to find image-regions and add the appropriate definition. By adding a co-attention system, we solve these problems, which simultaneously attends to images and projected tags and explores the synergistic effects of visual and semantic details.

Third, the explanations are typically lengthy in picture files, comprising several sentences. It's extremely nontrivial to produce such long file. Instead of implementing a single layer LSTM which is less capable of modeling long word series, we exploit the report's compositional character and

implement a hierarchical LSTM to generate long texts. The hierarchical LSTM, together with the co-attention system, creates high-level topics first, and then provides fine-grained explanations according to the topics.

Overall, the main contributions of our work are:

- We propose a multi-task learning framework that can predict the tags and produce text descriptions at the same time.

- We implement a system of co-attention for identifying subregions in the picture and producing the corresponding descriptions.

-We create a LSTM hierarchy for generating long paragraphs.

- We conduct detailed studies to show the effectiveness of the proposed methods.

\section{DESIGN PROCESS}

\section{A. Input Design}

Input architecture is the method of translating inputs derived from the consumer to a computer-based format. Input architecture is one of the most costly stages of computerized device activity and is always the main challenge of a program In the project, the specification of the data is rendered in various window types using specific techniques. For example, the empty username and password are not permitted in the user information section. If the username remains in the database, the entry is considered null and would not be acknowledged. Likewise, the username is a must during the authentication process, and must be included in the account

user list. Only logging in is then enabled. Input forms are:

- Login

- Add patient details

- Capture image

\section{B. Output Design}

Output architecture usually refers to the outcomes and knowledge produced by the program for other end-users; performance is the key justification for program implementation and the foundation in which the application's utility is evaluated.

Scan, in the user information, are the online types where the performance is accessible. Outputs are:

- Patient database

- Patient report

- Ultrasound images

\section{RESULT AND DISCUSSION}

\section{A. Admin Login}

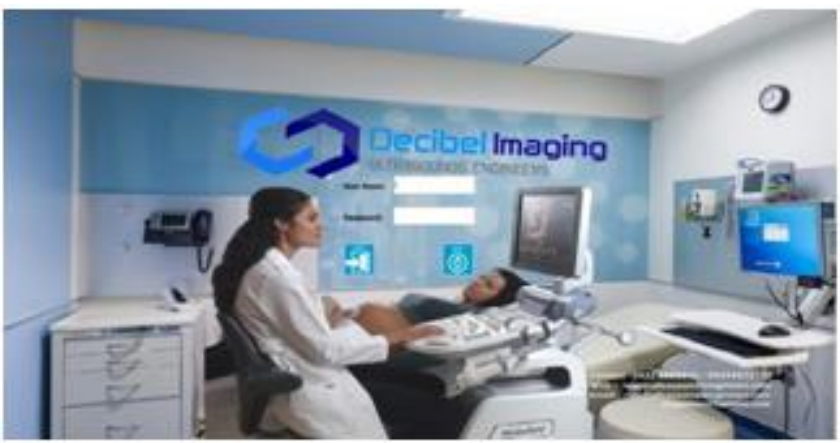

Fig. 2. Admin Login

\section{B. Patient Registration}

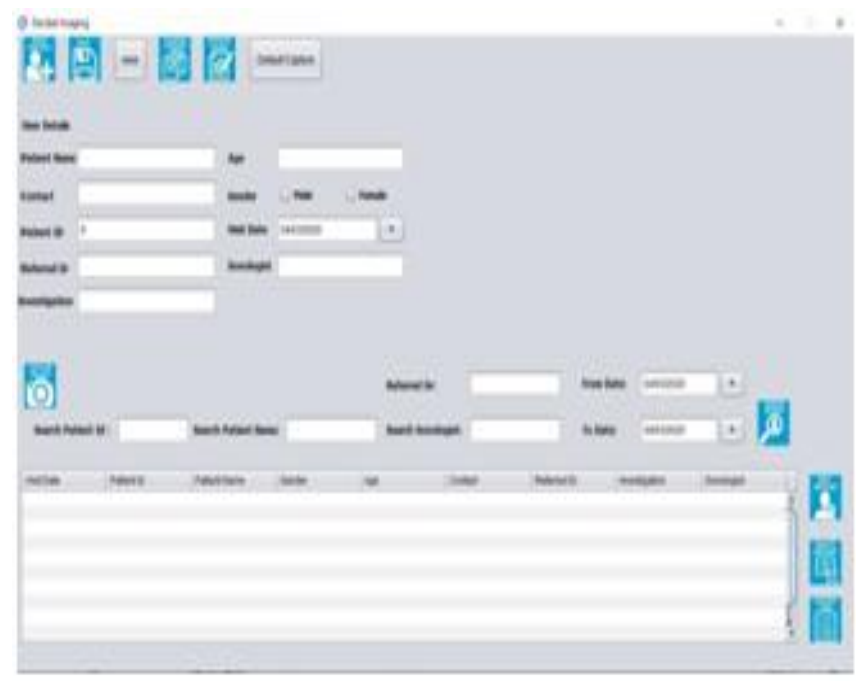

Fig. 3. Registration

Published By:

Blue Eyes Intelligence Engineering \& Sciences Publication

(C) Copyright: All rights reserved. 


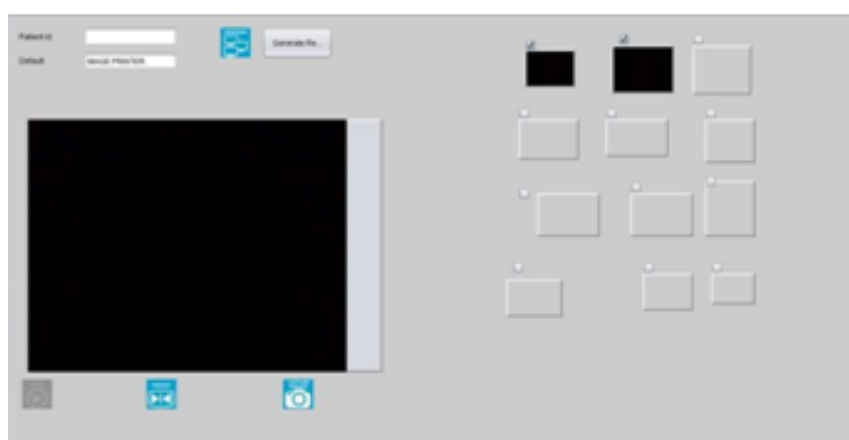

Fig. 4. Transaction

\section{Report Generation}
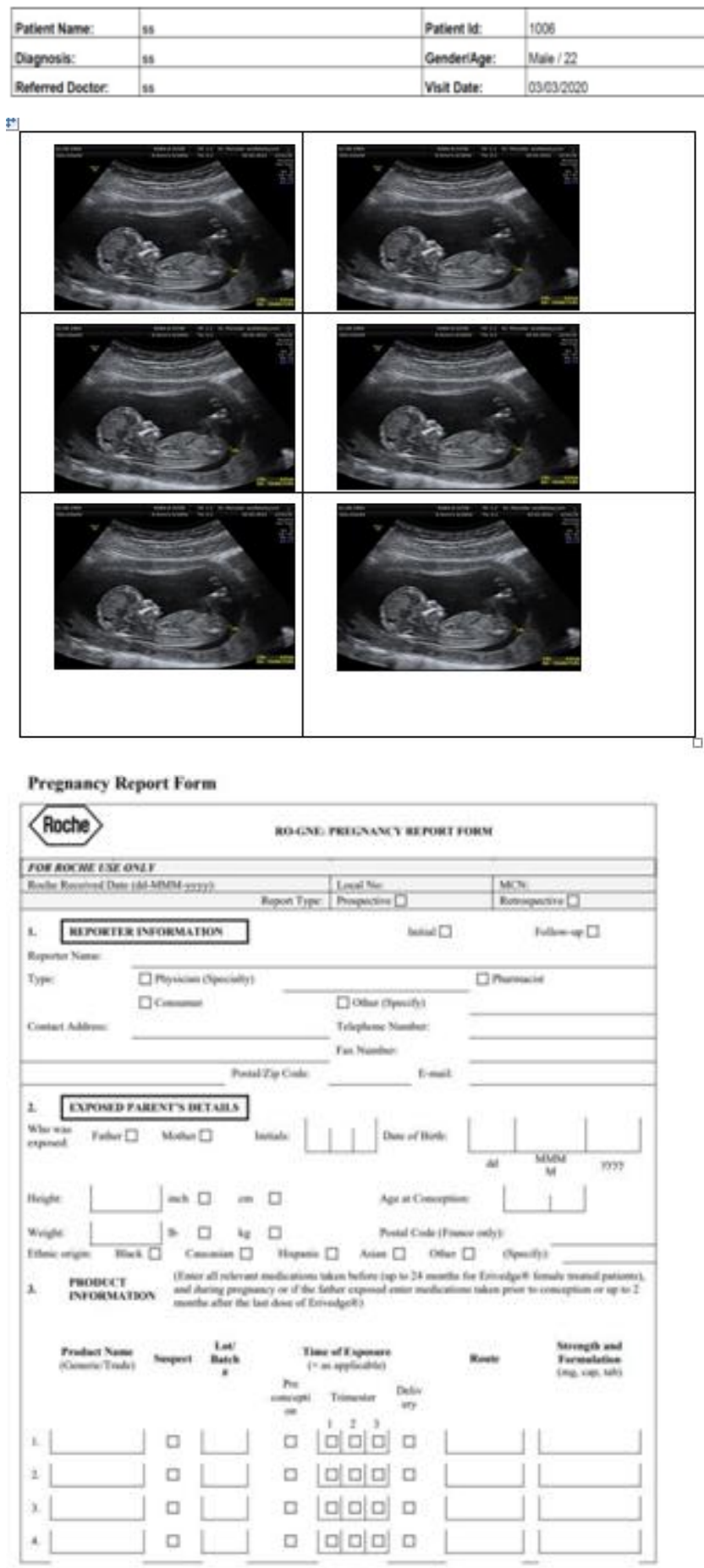
Arnold publishers Ltd, UK

\section{A. Conclusion}

Ultrasound imaging technique is the most reliable medical diagnosis procedure available. While the method is the best it needs speckle noise. This noise has many kinds of patterns that rely on certain imaging parameters. It is necessary to reduce the speckle noise produced by the imagine system when obtaining the ultrasonic images to enhance human perceptibility.

Usually despeckling is the basic stage in the production of medical image activities such as image recording, image enhancement; image reconstruction, etc. The effective approach for speckling noise reduction with edges protection was still a challenging job

It's very fascinating to see that while this topic is given too much focus, the problem remains open; various methods may allow any sort of changes in picture quality, But there was no particular approach that could be used to boost its efficiency on a standardized Ultrasonogram. The techniques offer only minor changes, too. As a consequence of the study, the future reach of work to develop improved algorithms correlating the principles of Ultrasound picture creation and modern Virtual image processing techniques is becoming apparent.

\section{B. Future Enhancement}

The prospect of ultrasound appears auspicious, such scans will be done in real time using 5-D ultrasound systems. The ultimate aim is to promote screening by better quality real-time imaging of the individual organs. The patient undergoes screening in the medical archive where the photographs are kept. The sonologist uses the Ultrasound test on the patient in intra-scan.

The generated ultrasound photographs are fused with the pre-existing dataset in real time and the output is constantly reflected on the computer, It provides the of complex and deformable merged artifacts with distinguishing features which can significantly aid to identify those regions and to make important decisions.

\section{REFERENCES}

1. David Sutton, "Radiology and Imaging for Medical Students", 7th Edition. Churchill Livingstone.

2. Bailey and Love's, "Short Practice of Surgery", 25th Edition. Edward

3. Richard N Czerwinski, Douglas. L. Jones and William D Brien, "Ultrasound speckle Reduction by Directional Median filtering", 1995

4. Latha Parthiban, R.Subramanian, "Speckle Noise Removal Using Contourlets", 2nd International Conference on Information and Automation, New Delhi, India 2006,

5. T Loupas, W N Mcdicken \& P L Allan, "An Adaptive Weighted Median filter for Speckle Suppression in medical ultra sonic images", IEEE Transactions on circuits and systems vol. 36, No 1, 1989.

6. K Thangavel, R Manavalan, I Laurence Aroquiaraj, "Removal of Speckle Noise from Ultrasound Medical Image based on Special Filters: Comparative Study", ICGST-GVIP Journal, ISSN $1687-$ 398X, Volume (9), Issue (III), 2009.

Jakia Afruz, Va'Juanna Wilson, Scott E Umbaugh, "Frequency Domain Pseudo-color to Enhance Ultrasound Images", Computer and Information Science Vol. 3, No. 4, 2010.

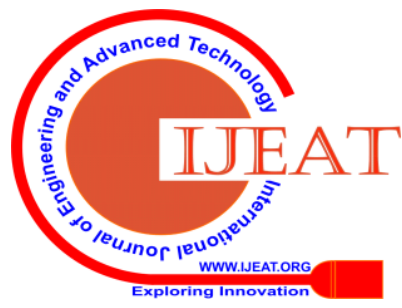


8. Savita Gupta, L Kaur, R C Chauhan and S C Saxena, "A Wavelet Based Statistical Approach for Speckle Reduction in Medical ultrasound Images", TENCON 2003, Conference on convergent Technologies for Asia-Pecific Region, Vol.2, pp 534-537, 2003

9. Zhao Chunhorg, Liu Mingyu \& Ji Hongbing "A new speckle reduction method of medical ultrasonic image", The 1st InternationalConference on Bioinformatics and Biomedical Engineering ICBBE, pp10041006, 2007

10. Chedsada Chinrungrueng, Aimamorn Suvichakorn, "Fast EdgePreserving Noise Reduction for Ultrasound Images", IEEE Transactions on Nuclear Science, Vol. 48, No. 3, 2001.

\section{AUTHORS PROFILE}

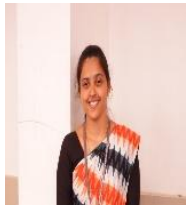

Sathya N, M.E working as assistant professor in department of Information Technology in Sri Shakthi Institute of Engineering and Technology. Research area: Machine learning Publication details: published about four research paper in International Journals and two papers in International Conference.

Email: nsathyait@siet.ac.in

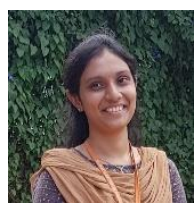

Swathi R, currently pursuing final year B Tech IT in Sri Shakthi Institute of Engineering and Technology, Coimbatore.

Email: swathirmkd@gmail.com

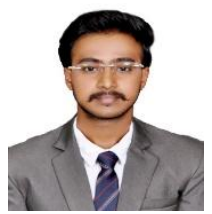

Sanjai D, currently pursuing final year B Tech IT in Sri Shakthi Institute of Engineering and Technology, Coimbatore.

Email: sanju29111998@gmail.com

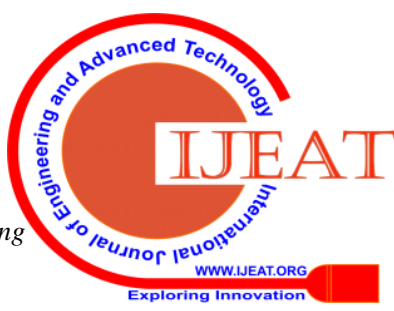

